Int. J. Contemp. Math. Sci., Vol. 2, 2007, no. 14, 679 - 683

\title{
A Result on Diffuse Random Measure
}

\author{
A. Varsei and H. Samimi \\ Department of Mathematics \\ Faculty of Sciences, The University of Guilan \\ P.O. Box 1914 P.C. 41938, Rasht, Iran \\ varsei@guilan.ac.ir, samimi@guilan.ac.ir
}

\begin{abstract}
We prove a result for a diffuse random measure with Radon intensity, from which it follows that if $\phi$ and $\psi$ are two random measures in $\Gamma$ defined on $\left(\mathfrak{M}^{(d)}, \mathcal{M}^{(d)}, P\right)$ such that

$\mathrm{E}\left(\phi(I) \mid \mathcal{M}_{I^{c}}^{(d)}\right)=\mathrm{E}\left(\psi(I) \mid \mathcal{M}_{I^{c}}^{(d)}\right) \mathrm{P}-$ a.s.

for all $\mathrm{I}$ in the union of a system of a partitions of $\Gamma$, then $\phi=\psi \mathrm{P}$ a.s.
\end{abstract}

Keywords: random measure, diffuse, intensity, conditional, Radon

\section{Introduction}

Let $\Gamma$ be a locally compact second countable Hausdorff topological space, i.e. a polish space, $\mathfrak{B}$ the $\sigma$-algebra of Borel subsets of $\Gamma, \mathfrak{B}_{0}$ the family of bounded sets in $\mathfrak{B}$ and let $\mathfrak{M}$ be the space of non negative Borel measures which are finite on $\mathfrak{B}_{0}$, the space of Radon Measures, and $\mathcal{M}$ be the $\sigma$ algebra in $\mathfrak{M}$ generated by the mappings $\mu \rightarrow \mu(B), B \in \mathfrak{B}_{0}$, (i.e. smallest $\sigma$ - algebra making these mappings measurable) and $\mathfrak{M}^{(d)}$ denotes the subset of $\mathfrak{M}$ consisting of all diffuse measures and $\mathcal{M}^{(d)}=\mathcal{M} \cap \mathfrak{M}^{(d)}$. For $I \in \mathfrak{B}$, $\mathcal{M}_{I}$ denotes the $\sigma$ - algebra in $\mathfrak{M}$ generated by mappings $\mu \rightarrow \mu(B), B \in$ $\mathfrak{B}_{0}, B \subseteq I . \mathcal{M}_{I}^{(d)}$ is defined similarly for any $I \in \mathfrak{B}$. A random measure in $\Gamma$ is a probability measure on $(\mathfrak{M}, \mathcal{M})$ or a measurable function from some probability space into $(\mathfrak{M}, \mathcal{M})$. Diffuse random measure is defined similarly. If $\xi$ is a random measure, it is clear that for fixed $I \in \mathfrak{B}_{0}$, the mapping $\omega \rightarrow \xi(\omega, I)$ is a random variable, which will be denoted by $\xi(I)$. Also $\xi^{(d)}(I)$ denotes the restriction of $\xi(I)$ on $\mathfrak{M}^{(d)}$.For a Radon measure $\omega$ in $\Gamma$ and any $I \in \mathfrak{B}$, define $\bar{\xi}(\omega, I)=\omega(I)$. If $\mathrm{P}$ is a probability measure on $(\mathfrak{M}, \mathcal{M})$, the measure in $\Gamma$ given by $\mathrm{E}(\bar{\xi}(I))=\int \bar{\xi}(\omega, I) P(d \omega), I \in \mathfrak{B}$, is called the intensity of P. Similarly if $\xi$ is a measurable function from some probability space into 
$(\mathfrak{M}, \mathcal{M})$, the measure in $\Gamma$ given by $\mathrm{E}(\xi(I))$ is called the intensity of $\xi$. Finally a system of partitions of $\Gamma$ (compare with null array of partitions in [3] and see [1]) means a sequence $\left\{\Delta_{n}\right\}_{n \geq 1}$ of partitions of $\Gamma$ having the following properties:

1. Each $\Delta_{n}$ consists of countable many bounded Borel sets.

2. $\Delta_{n+1}$ is a refinement of $\Delta_{n}$ for all $n \geq 1$.

3. For any $\Gamma^{\prime} \subseteq \Gamma, \Gamma^{\prime} \in \mathfrak{B}_{0}$, and any $\varepsilon>0$, there is some $n \geq 1$, such that $\Gamma^{\prime}$ can be covered by a finite number of elements of $\Delta_{n}$ each having diameter smaller than $\varepsilon$.

The topological structure of $\Gamma$ ensures the existence of such a sequence of partitions (see [2]).

In this article we prove that if $\phi$ and $\psi$ are two random measure in $\Gamma$ defined on

$\left(\mathfrak{M}^{(d)}, \mathcal{M}^{(d)}, \mathrm{P}\right)$, with Radon intensity, such that

$$
\mathrm{E}\left(\phi(I) \mid \mathcal{M}_{I^{c}}^{(d)}\right)=\mathrm{E}\left(\psi(I) \mid \mathcal{M}_{I^{c}}^{(d)}\right) \mathrm{P}-\text { a.s. }
$$

for all $I \in \bigcup_{n \geq 1} \Delta_{n}$, where $I^{c}$ represents the complement of $I$, then $\phi=\psi$ P- a.s. (partially case was done in [5] ).

\section{Main Theorem}

We first represent the following lemmas.

Lemma 1 . If $\phi$ and $\psi$ are two random measures in $\Gamma$ on $\left(\mathfrak{M}^{(d)}, \mathcal{M}^{(d)}, P\right)$ and satisfying (1), for any $I \in \bigcup_{n \geq 1} \Delta_{n}$ and disjoint $I_{1}, I_{2}, \ldots, I_{r} \in \bigcup_{n \geq 1} \Delta_{n}$ and any $a_{1}, a_{2}, \ldots, a_{r} \geq 0$ :

$$
\int_{H} \phi(\omega, I) P(d \omega)=\int_{H} \psi(\omega, I) P(d \omega)
$$

Where $H=\left\{\bar{\xi}\left(I_{1}\right) \leq a_{1}, \ldots, \bar{\xi}\left(I_{r}\right) \leq a_{r}\right\}$.

Lemma 2 . Two finite measures $\mu_{1}$ and $\mu_{2}$ on $\left(\mathfrak{M}^{(d)}, \mathcal{M}^{(d)}\right)$ with $\mu_{1}\left(\mathfrak{M}^{(d)}\right.$ )$=\mu_{2}\left(\mathfrak{M}^{(d)}\right)$ are identical if, and only if, for any disjoint $I_{1}, I_{2}, \ldots, I_{r} \in \bigcup_{n \geq 1} \Delta_{n}$ ,$\left(\bar{\xi}\left(I_{1}\right)_{1}, \ldots, \bar{\xi}\left(I_{r}\right)\right)$ maps $\mu_{1}$ and $\mu_{2}$ to the same measure in $[0,+\infty)^{r}$. Theorem

If $\phi$ and $\psi$ are two random measures in $\Gamma$ defined on $\left(\mathfrak{M}^{(d)}, \mathcal{M}^{(d)}, \mathrm{P}\right), \phi, \psi$ have Radon intensity,

such that $\mathrm{E}\left(\phi(I) \mid \mathcal{M}_{I^{c}}^{(d)}\right)=\mathrm{E}\left(\psi(I) \mid \mathcal{M}_{I^{c}}^{(d)}\right) \mathrm{P}-$ a.s.

for all $\mathrm{I} \in \bigcup_{n \geq 1} \Delta_{n}$, then $\phi=\psi$ P- a.s. 


\section{Proof.}

For fixed $\mathrm{I} \in \bigcup_{n \geq 1} \Delta_{n}$, define a measure on $\mathcal{M}^{(d)}$ by

$\mu_{1}(A)=\int_{A} \phi(\omega, I) P(d \omega), A \in \mathcal{M}^{(d)}$,

and one by

$\mu_{2}(A)=\int_{A} \psi(\omega, I) P(d \omega), A \in \mathcal{M}^{(d)}$.

Relation (2), in view of lemma 2 , clearly implies that $\mu_{1}=\mu_{2}$, i.e.

$$
\int_{A} \phi(\omega, I) P(d \omega)=\int_{A} \psi(\omega, I) P(d \omega)
$$

for all $A \in \mathcal{M}^{(d)}$. This in turn implies that for P- a.e. $\omega$,

$$
\phi(\omega, I)=\psi(\omega, I)
$$

Since $\bigcup_{n \geq 1} \Delta_{n}$ is countable, it follows that there is a P-null event $N$ such that if $\omega \notin N$, then

$$
\phi(\omega, S)=\psi(\omega, S)
$$

for all $S \in \bigcup_{n \geq 1} \Delta_{n}$ and therefore $S \in \mathrm{B}$.

The proof of theorem is thus complete.

\section{Proof of the lemmas}

\section{Proof of lemma 1:}

Suppose $I, I_{0} \in \bigcup_{n \geq 1} \Delta_{n}$ with $I \subseteq I_{0}$, and $a \geq 0, A \in \mathcal{M}_{I_{0}^{c}}^{(d)}$, are fixed. Define, for $n \geq \nu(I),(\nu(I)$ denotes the smallest positive integer $\mathrm{n}$ such that $\mathrm{I}$ can be expressed as a union of elements of $\Delta_{n}$ )

$$
X_{n}(\omega)=\sum_{J \in \Delta_{n}^{(I)}} \chi_{\left\{\bar{\xi}\left(I_{0} \backslash J\right) \leq a\right\}}(\omega) \phi(\omega, J)
$$

Where $\Delta_{n}^{(I)}$ denotes the family $\left\{J \in \Delta_{n}: J \subseteq I\right\}$.

For each $\omega \in \mathfrak{M}^{(d)}$, on account of its diffuseness,

$$
\lim _{n \rightarrow \infty} \max _{J \in \Delta_{n}^{(I)}} \bar{\xi}(\omega, J)=0
$$


Therefore

$$
\lim _{n \rightarrow \infty} X_{n}(\omega)=\chi_{\left\{\bar{\xi}\left(I_{0}\right) \leq a\right\}}(\omega) \phi(\omega, I) .
$$

Since $X_{n}(\omega) \leq \phi(\omega, I)$, from the dominated convergence theorem it follows that

$$
\lim _{n \rightarrow \infty} E\left(\chi_{A} X_{n}\right)=E\left(\chi_{A \cap\left\{\bar{\xi}\left(I_{0}\right) \leq a\right\}} \phi(I)\right) .
$$

If we define, for $n \geq \nu(I)$,

$$
Y_{n}(\omega)=\sum_{J \in \Delta_{n}^{(I)}} \chi_{\left\{\bar{\xi}\left(I_{0} \backslash J\right) \leq a\right\}}(\omega) \psi(\omega, J),
$$

It follows similarly that

$$
\lim _{n \rightarrow \infty} E\left(\chi_{A} Y_{n}\right)=E\left(\chi_{A \cap\left\{\bar{\xi}\left(I_{0}\right) \leq a\right\}} \psi(I)\right) .
$$

For each $n \geq \nu(I)$, in view of (1) we have

$$
E\left(\chi_{A} X_{n}\right)=E\left(\chi_{A} Y_{n}\right)
$$

It therefore follows that

$$
E\left(\chi_{A \cap\left\{\bar{\xi}\left(I_{0}\right) \leq a\right\}} \phi(I)\right)=E\left(\chi_{A \cap\left\{\bar{\xi}\left(I_{0}\right) \leq a\right\}} \psi(I)\right) .
$$

Now, if $I_{1}, I_{2}, \ldots, I_{r} \in \bigcup_{n \geq 1} \Delta_{n}$ are disjoint, it is not difficult to see that we can choose $n_{0}$ such that for each $J \in \Delta_{n_{0}}^{(I)}$, either $J \subseteq I_{s}$ for some $s, 1 \leq s \leq r$ , or $J \subseteq \Gamma \backslash\left(I_{1} \cup \ldots \cup I_{r}\right)$.

Then

$$
\begin{gathered}
\int_{H} \phi(\omega, I) P(d \omega) \\
=\sum_{J \in \Delta_{n_{0}}^{(I)}, J \subseteq \bigcup I_{s}} \int_{H} \phi(\omega, J) P(d \omega)+\sum_{J \in \Delta_{n_{0}}^{(I)}, J \subseteq \Gamma \backslash\left(\bigcup I_{s}\right)} \int_{H} \phi(\omega, J) P(d \omega)
\end{gathered}
$$




$$
=\sum_{J \in \Delta_{n_{0}}^{(I)}, J \subseteq \bigcup I_{s}} \int_{H} \psi(\omega, J) P(d \omega)+\sum_{J \in \Delta_{n_{0}}^{(I)}, J \subseteq \Gamma \backslash\left(\bigcup I_{s}\right)} \int_{H} \psi(\omega, J) P(d \omega)
$$

from (3) and (1), respectively,

$$
=\int_{H} \psi(\omega, I) P(d \omega)
$$

\section{Proof of lemma 2:}

This lemma is a consequence of the following lemma which is consequence of lemma I.2.1 and Theorem 3.1 of [3].

\section{Lemma 3:}

Let $\phi$ and $\psi$ be two random measures in $\Gamma$ (two measurable functions from some probability space into $(\mathfrak{M}, \mathcal{M})) . \phi$ and $\psi$ have the same distribution in $\mathfrak{M}$ if, and only if, for any disjoint $I_{1}, I_{2}, \ldots, I_{r} \in \bigcup_{n \geq 1} \Delta_{n}$, the random vectors $\left(\phi\left(I_{1}\right), \ldots, \phi\left(I_{r}\right)\right)$ and $\left(\psi\left(I_{1}\right), \ldots, \psi\left(I_{r}\right)\right)$ are identically distributed .

\section{References}

[1] P. Jacob, P.E. Oliveira, On the conditional intensity of a random measure 35,1 (1994) 103-109.

[2] O. Kallenberg, On conditional intensities of point processes.Z. Wahrsch verw .Geb.41 (1987) 205-220.

[3] O. Kallenberg, Random measure, Academic Press, 1983.

[4] F. Papangelou., The conditional intensity of general point processes and an application to line processes, Z. Wahrsch verw.Geb.28(1974) 207-226.

[5] F. Papangelou., Point processes on spacees of flats and other homogeneous Spaces, Math. Proc. Cambridge Pill.Soc. 80 (1976), 297-314.

[6] S.I. Resink, A probability path, Birkhauser Boston, 2001.

\section{Received: August 8, 2006}

